\title{
Qualidade de vida e fatores associados na diabetes mellitus tipo 2: estudo observacional
}

Soraia Santos, ${ }^{*}$ Helena Beça, ${ }^{* *}$ Carla Lopes da Mota***

\section{RESUMO}

Introdução: A diabetes mellitus (DM) tipo 2 é uma doença crónica com elevados custos sociais e económicos. Está frequentemente associada à diminuição da qualidade de vida (QoL).

Objetivos: Perceber o impacto de alguns fatores sociodemográficos e clínicos na QoL numa amostra de doentes com DM tipo 2. Tipo de estudo: Observacional, transversal e analítico.

Local: USF Espinho.

População: Constituída por 572 utentes com DM tipo 2 vigiados na USF Espinho, em dezembro de 2012.

Métodos: Utilizou-se uma amostra de conveniência, constituída por 231 utentes que se dirigiram à USF para uma consulta de vigilância de DM. Foram recolhidas informações sociodemográficas e clínicas e foi aplicado o questionário Perfil de Saúde do Diabético (DHP) para avaliação da QoL. A influência das variáveis contínuas nos resultados das dimensões do DHP avaliou-se com o coeficiente de correlação de Pearson e a influência das variáveis categóricas com os testes $t$-student e ANOVA.

Resultados: O género feminino, menos habilitações literárias, hipoglicemia severa e complicações microvasculares apresentaram um impacto negativo na dimensão "tensão psicológica" da QoL. As "barreiras à atividade" foram negativamente afetadas por insulinoterapia e hipoglicemia severa. Os doentes com menos habilitações literárias e os não controlados do ponto de vista glicémico revelaram menor QoL no domínio "alimentação desinibida".

Conclusões: A QoL é um conceito complexo com múltiplos fatores determinantes. É importante o estudo destes fatores e a sua avaliação em cada doente com DM para uma intervenção mais eficaz.

Palavras-chave: Diabetes Mellitus Tipo 2; Qualidade de Vida.

\section{INTRODUÇÃO}

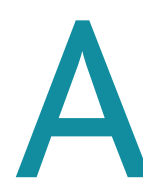

diabetes mellitus (DM) é uma doença que acarreta custos humanos, sociais e económicos elevados. Estimativas recentes da International Diabetes Federation indicam que esta doença afeta aproximadamente 382 milhões de pessoas, cerca de $8,3 \%$ da população mundial. ${ }^{1}$ Calcu-

\footnotetext{
* Mestrado Integrado em Medicina, Faculdade de Medicina da Universidade do Minho. Médica Interna do Ano Comum, no Centro Hospitalar Entre Douro e Vouga **Médica Assistente de Medicina Geral e Familiar, na Unidade de Saúde Familiar Espinho (ACeS Espinho/Gaia)

***Médica Assistente de Medicina Geral e Familiar, na Unidade de Cuidados de Saúde Personalizados Barão do Corvo (ACeS Gaia)
}

la-se que a DM tipo 2 represente 85 a $95 \%$ dos casos de diabetes em países desenvolvidos. ${ }^{1}$ Em Portugal, o Observatório Nacional da Diabetes (OND) estimou, para 2013, uma prevalência desta doença de $13 \%$ na população entre os 20 e os 79 anos, representando um total de 8-9\% das despesas em saúde nesse ano. ${ }^{2}$ Segundo o mesmo organismo, foi responsável por 4.867 mortes em 2012. ${ }^{2}$

Geralmente os doentes valorizam mais o impacto do que a doença, a medicação e os procedimentos médicos no seu estilo de vida do que os marcadores clínicos da mesma. ${ }^{3}$ Por isso, nos últimos anos, a qualidade de vida (QoL) - definida pela Organização Mundial 
da Saúde como "a perceção do indivíduo da sua posição na vida no contexto da cultura e sistemas de valores nos quais ele vive e em relação aos seus objetivos, expectativas, padrões e preocupações" ${ }^{\prime \prime}$ assumiu-se como um dos principais objetivos da gestão das doenças. ${ }^{5}$

Nas pessoas com diabetes, pior QoL tem sido associada a tratamento com insulina ${ }^{6-8}$ descontrolo glicémico, ${ }^{5,9}$ hipoglicemia, ${ }^{6,10}$ complicações crónicas ${ }^{5-6,8} \mathrm{e}$ aumento da mortalidade. ${ }^{11}$ Não parece haver uma relação entre a idade e a diabetes no que diz respeito à QoL, já que o aumento da idade se relaciona com melhor QoL na componente de saúde mental e menor na componente de saúde física da mesma forma em doentes com ou sem DM tipo 2.12 A escolaridade está associada a melhor QoL..$^{5-6}$ Em Portugal existem estudos que avaliam os fatores determinantes da QoL de doentes com DM tipo $1,,^{13-14}$ mas são escassos quanto à DM tipo $2^{15-16} \mathrm{e} \mathrm{com}$ pouco ênfase nos fatores sociodemográficos ou clínicos que a influenciam.

Uma vez que a identificação das determinantes da QoL é o primeiro passo para minimizar o impacto negativo da doença no dia-a-dia, pretende-se com este estudo caracterizar a QoL das pessoas com diabetes tipo 2 , verificar se existe associação com características sociodemográficas e clínicas e identificar os fatores com maior impacto na mesma.

\section{MÉTODOS}

Foi realizado um estudo observacional, transversal e analítico, na USF Espinho, de julho a dezembro de 2013. A população era constituída pelos utentes com diagnóstico de DM tipo 2 vigiados na USF Espinho em dezembro de 2012 ( $n=572$ ). Foram excluídos os doentes incapazes de ler e responder ao questionário e sem pelo menos um valor de HbAlc registado no ano de 2013 (utilizado para definir o controlo glicémico).

Utilizou-se uma amostra de conveniência, constituída por 231 utentes, que se dirigiram à USF Espinho para uma consulta de vigilância de DM, entre agosto e outubro de 2013. Os utentes foram convidados a colaborar no estudo pelo médico de família e depois encaminhados para a investigadora responsável pela colheita dos dados. Aos que acederam participar foi explicado o estudo e solicitado, pela mesma investigadora, a assinatura do consentimento informado.
Para a avaliação da QoL utilizou-se um instrumento específico, o Perfil de Saúde do Diabético (DHP), validado para a população portuguesa, ${ }^{17}$ que mede a disfunção psicossocial e comportamental em diabéticos. A sua avaliação divide-se em três dimensões: "tensão psicológica", "barreiras à atividade" e "alimentação desinibida", sendo cada uma classificada numa escala de 0 a 100, em que valores mais altos representam pior QoL.

Adicionalmente, foram recolhidos por entrevista dados sociodemográficos (data de nascimento, idade, género, estado civil e habilitações literárias) e averiguado se ocorreu episódio de hipoglicemia severa no último ano (episódio com necessidade de intervenção de outra pessoa para administração de hidratos de carbono, glucagon ou aplicação de outras medidas de reanimação).$^{18} \mathrm{O}$ acesso ao dados clínicos [duração da doença determinada a partir da data de diagnóstico de DM, regime terapêutico (nenhum fármaco, antidiabéticos orais, insulina), número de antidiabéticos orais, tipo de controlo glicémico (se HbAlc $<7 \%$ - controlado; se HbAlc $\geq 7 \%$ - não controlado) ${ }^{19}$ complicações macrovasculares (doença cerebrovascular, doença cardiovascular, doença arterial periférica $)^{20}$ e complicações microvasculares (retinopatia, neuropatia e nefropatia ${ }^{20}$ ] ocorreu através de consulta do processo clínico eletrónico - sistema de apoio ao médico (SAM). Estas complicações foram definidas de acordo com a codificação da International Classification Primary Care $-2^{\text {nd }}$ edition (ICPC-2), como se pode observar na Figura 1. Relativamente às complicações microvasculares, existe na ICPC-2 uma codificação específica para a nefropatia associada à DM (Glomerulonefrite/Nefrose - U88), sendo esta a que é codificada quando é assinalado no Programa de Diabetes do SAM a presença de nefropatia. Assim, determinou-se estar presente esta complicação nos doentes com registo de U88 - Glomerulonefrite/Nefrose nos antecedentes pessoais. Não se considerou a codificação de U99 Outras doenças urinárias e que inclui a insuficência renal crónica atendendo às múltiplas causas desta patologia, não exclusiva da DM.

Posteriormente foi entregue o questionário DHP, preenchido pelo utente, sempre na presença da mesma investigadora. Esta verificava se o questionário estava integralmente preenchido, solicitando ao utente a 


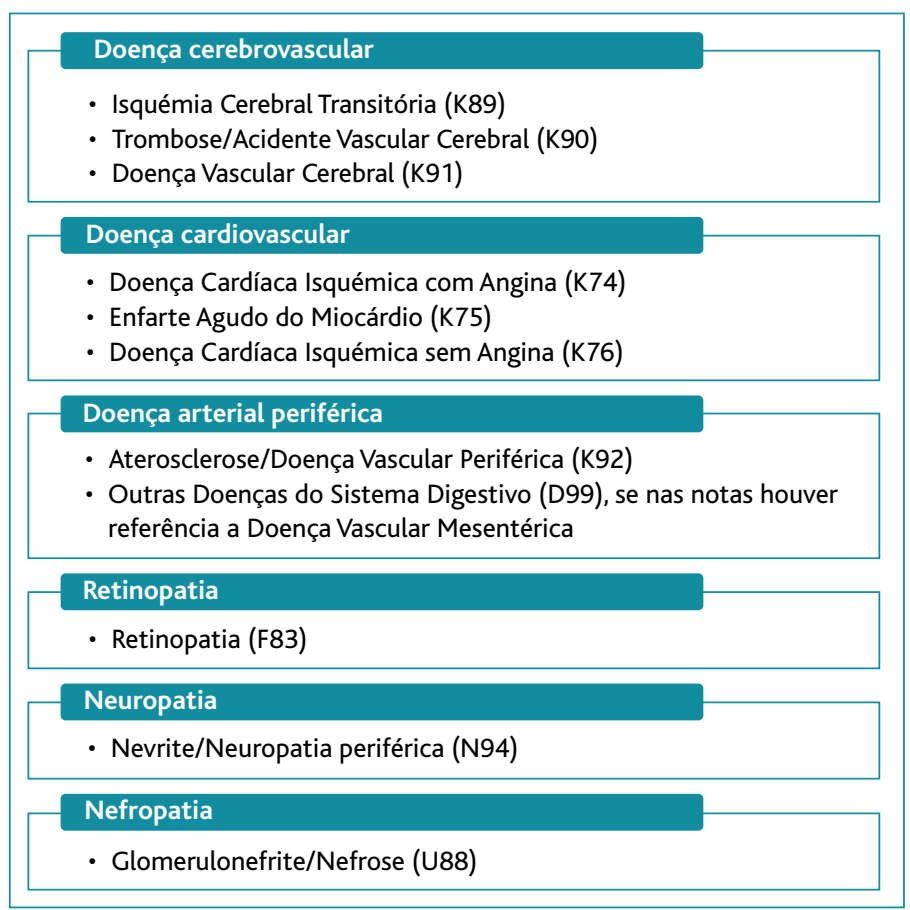

Figura 1. Definição das complicações macro e microvasculares, segundo a ICPC-2.

sua conclusão sempre que necessário.

Elaborou-se uma base de dados eletrónica e recorreu-se ao programa informático Statistical Package for the Social Science ${ }^{\circledast}$, versão 22, para tratamento dos dados. A existência de distribuição normal foi avaliada através de valores de assimetria e curtose (considerou-se como distribuição normal valores entre 2 e -2) e de histogramas. $^{21}$

Para avaliar a influência das variáveis contínuas nos resultados das dimensões do DHP determinou-se o coeficiente de correlação de Pearson. Para as variáveis categóricas realizou-se o teste de Levene para a igualdade de variâncias e foram utilizados os testes $t$-student e ANOVA. Quando encontradas diferenças estatisticamente significativas após aplicação do teste ANOVA foi realizado o teste post-hoc Tukey. Para os testes com diferenças estatisticamente significativas calculou-se a magnitude de efeito através do $d$ de Cohen para o $t$-stu$\operatorname{dent}$ (pequeno se $0,20 \leq d<0,50$, médio se $0,50 \leq d<0,80$ e grande se $d \geq 0,80$ ) e do $\eta^{2}$ para a ANOVA (pequeno se $0,01 \leq \eta^{2}<0,06$, médio se $0,06 \leq \eta^{2}<0,14$ e grande se $\eta^{2}$ $\geq 0,14) .{ }^{22}$
Para os testes de hipóteses, devido à desigualdade numérica existente entre os grupos nas variáveis estado civil, habilitações literárias, regime terapêutico e número de antidiabéticos, procedeu-se à agregação dos existentes, formando novos grupos com menores diferenças de tamanho entre si. Na variável estado civil formaram-se dois grupos, consoante a existência (casado/a e união de facto) ou não (solteiro/a, viúvo/a e divorciado/a) de relação conjugal. Agruparam-se os grupos das habilitações literárias em três: escolaridade inferior ao $1 .^{\circ}$ ciclo, $1 .^{\circ}$ ciclo de escolaridade e escolaridade superior ao $1 .{ }^{\circ}$ ciclo. Atendendo à influência da insulinoterapia na QoL reportada na bibliografia, dividiu-se a variável regime terapêutico em dois grupos, sem insulinoterapia e com insulinoterapia. Finalmente, porque a maioria dos doentes realizava apenas um antidiabético oral, para uniformizar o tamanho dos grupos agregaram-se os existentes, passando a variável regime terapêutico a ser constituída pelo grupo que realizava nenhum ou apenas um fármaco e pelo grupo cuja terapêutica era constituída por mais de um fármaco.

Todas as variáveis que apresentaram relação estatisticamente significativa com pelo menos uma dimensão do DHP foram inseridas num modelo de regressão linear múltipla. Foi utilizado um nível de significância de 0,05 para toda a análise estatística.

O questionário DHP foi aplicado após autorização dos autores da versão validada para a população portuguesa.

Foram obtidos pareceres favoráveis do Coordenador da USF Espinho, do Diretor Executivo do Agrupamento de Centros de Saúde Espinho/Gaia, da Comissão de Ética para a Saúde da Administração Regional de Saúde do Norte e da Subcomissão de Ética para as Ciências da Vida e da Saúde da Universidade do Minho.

\section{RESULTADOS}

Responderam ao questionário 231 doentes diabéticos.

Todas as variáveis contínuas apresentaram distribuição normal.

Como se pode observar no Quadro I, a média de ida- 


\begin{tabular}{|c|c|}
\hline \multicolumn{2}{|c|}{$\begin{array}{l}\text { QUADRO I. Caracterização sociodemográfica e clínica } \\
\text { da amostra. }\end{array}$} \\
\hline Idade, $M(D P)$ & $67,5(9,3)$ \\
\hline Género, n (\%) & \\
\hline Masculino & $115(49,8)$ \\
\hline Feminino & $116(50,2)$ \\
\hline Estado civil, n (\%) & \\
\hline Solteiro/a & $18(7,8)$ \\
\hline Casado/a & $152(65,8)$ \\
\hline Viúvo/a & $48(20,8)$ \\
\hline Divorciado/a & $12(5,2)$ \\
\hline União de facto & $1(0,4)$ \\
\hline Habilitações literárias, n (\%) & \\
\hline$<1 .{ }^{\circ}$ ciclo & $43(18,6)$ \\
\hline 1. ${ }^{\circ}$ Ciclo & $107(46,3)$ \\
\hline 2. ${ }^{\circ}$ Ciclo & $20(8,7)$ \\
\hline 3. ${ }^{\circ}$ Ciclo & $25(10,8)$ \\
\hline Secundário & $14(6,1)$ \\
\hline Curso técnico-profissional & $10(4,3)$ \\
\hline Licenciatura/mestrado/doutoramento & $12(5,2)$ \\
\hline Duração de doença, $M(D P)$ & $10,4(8,5)$ \\
\hline Regime terapêutico, n (\%) & \\
\hline Sem tratamento farmacológico & $5(2,2)$ \\
\hline Antidiabéticos orais & $196(84,8)$ \\
\hline Insulinoterapia & $6(2,6)$ \\
\hline Antidiabéticos orais + insulinoterapia & $24(10,4)$ \\
\hline N. ${ }^{\circ}$ antidiabéticos orais, $n(\%)$ & \\
\hline 0 & $11(4,8)$ \\
\hline 1 & $131(56,7)$ \\
\hline 2 & $63(27,3)$ \\
\hline 3 & $23(10,0)$ \\
\hline$>3$ & $3(1,3)$ \\
\hline Controlo glicémico, n (\%) & \\
\hline Controlado & $156(67,5)$ \\
\hline Hipoglicemia severa último ano, n (\%) & \\
\hline Sim & $20(8,7)$ \\
\hline Complicações microvasculares, n (\%) & \\
\hline Sim & $33(14,3)$ \\
\hline Complicações macrovasculares, n (\%) & \\
\hline Sim & $40(17,3)$ \\
\hline Dimensões do DHP, M (DP) & \\
\hline "Tensão psicológica" & $20,0(12,5)$ \\
\hline "Barreiras à atividade" & $30,5(17,0)$ \\
\hline "Alimentação desinibida" & $27,7(18,3)$ \\
\hline
\end{tabular}

M-média

DP - desvio-padrão

$\mathrm{n}$ - frequência absoluta

$\%$ - frequência relativa

DHP - Diabetes Health Profile/Perfil de Saúde do Diabético

des foi de 67,5 anos, $49,8 \%$ da amostra eram homens, cerca de $66,0 \%$ dos indivíduos eram casados e $46,3 \%$ tinham o $1 .{ }^{\circ}$ ciclo.

Em relação às características clínicas (Quadro I), a duração da doença foi em média de 10,4 anos. A maioria dos doentes estava tratada apenas com antidiabéticos orais e, destes, $56,7 \%$ realizava apenas um fármaco. Cerca de $68 \%$ dos doentes encontravam-se controlados do ponto de vista glicémico. Relativamente às complicações, foi reportado pelo menos um episódio de hipoglicemia severa no último ano por 20 participantes $(8,7 \%)$ e $26,4 \%$ dos doentes tinham pelo menos uma complicação crónica.

Nas dimensões "tensão psicológica" e "barreiras à atividade", a média foi de 20,0 e 30,5, respetivamente. A média da "alimentação desinibida" foi de 27,7 (Quadro I).

A análise da correlação entre as três dimensões do questionário foi muito significativa entre todos os pares $(p<0,001)$. O maior coeficiente de correlação obtido foi entre "tensão psicológica" e "barreiras à atividade" $(r=0,62)$, seguida da "alimentação desinibida" e "tensão psicológica" $(r=0,44)$. A relação entre as dimensões "alimentação desinibida" e "barreiras à atividade" apresentou uma menor correlação $(r=0,28)$.

Não foram observadas diferenças estatisticamente significativas na idade, estado civil, número de antidiabéticos orais e complicações macrovasculares para as três dimensões do questionário (Quadro II).

Na dimensão "tensão psicológica", a média dos resultados obtidos pelo género feminino foi superior à do género masculino, sendo esta relação estatisticamente significativa $[t(224)=-3,68$; diferença média $(\mathrm{DFM})$ : -5,8; Intervalo Confiança (IC) 95\%: -9,0; -2,7] e com uma magnitude de efeito pequena $(d=0,42)$. Foram encontradas diferenças estatisticamente significativas entre os grupos das habilitações literárias $[F(2,228)=$ $6,82 ; p=0,001]$, com um efeito médio nesta dimensão $\left(\eta^{2}=0,06\right)$ (Quadro II), tendo sido posteriormente realizado um teste post-hoc que demonstrou que a média dos doentes com escolaridade inferior ao $1 .^{\circ}$ ciclo era superior, de forma estatisticamente significativa, à do grupo com escolaridade superior ao $10^{\circ}$ ciclo (DFM: $8,2$; IC $95 \%: 2,6 ; 13,7)$ e do $1 .{ }^{\circ}$ ciclo maior que o do grupo com escolaridade superior ao $1 .^{\circ}$ ciclo (DFM: 4,4; IC95\%: 0,1; 8,7), sem diferenças estatisticamente 


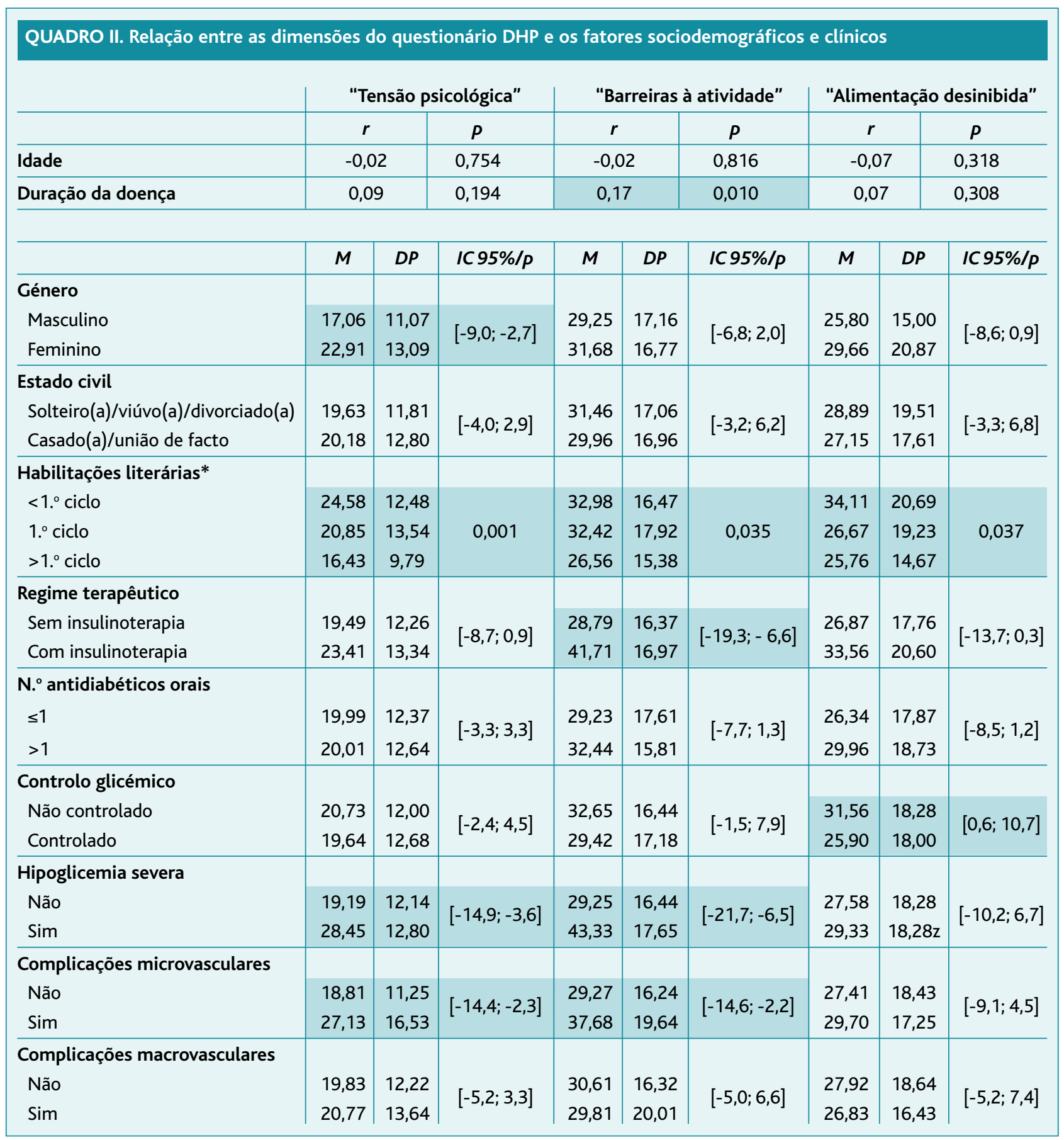

$r$ - Coeficiente de correlação de Pearson

$P$ - $P$-value; IC95\%: intervalo de confiança a 95\% para a diferença de médias

$M$ - média; $D P$ - desvio-padrão

* - IC95\% apresentado na secção resultados para cada comparação entre grupos

- Relação estatisticamente significativa

DHP - Diabetes Health Profile/Perfil de Saúde do Diabético 
significativas entre o outro par (escolaridade inferior ao $1 .^{\circ}$ ciclo versus $1 .^{\circ}$ ciclo - DFM: 3,7 ; IC95\%: $-1,5 ; 9,0$ ).

O score médio dos doentes que reportaram hipoglicemia severa foi superior à dos indivíduos que afirmaram não ter tido nenhum episódio $[t(229)=$ 3,24; DFM: -9,3; IC95\%: -14,9; -3,6], apresentando esta variável uma magnitude de efeito média $(d=0,74)$. Os doentes com complicações microvasculares apresentaram um score médio superior, estatisticamente significativo, aos doentes sem estas patologias $[t(37)=-2,79$; DFM: $-8,3$; IC95\%: $-14,4$; $2,3]$, com uma magnitude de efeito média $(d=0,66)$ (Quadro II). As restantes variáveis não demonstraram efeito estatisticamente significativo nesta dimensão.

Foi criado um modelo com as quatro variáveis com impacto na "tensão psicológica" e todas mantiveram uma relação estatisticamente significativa. O modelo criado explica $14 \%$ da variabilidade desta dimensão $\left[F(4,226)=10,14 ; p<0,001 ; R^{2}{ }_{a d j}=0,14\right]$ (Quadro III).

Dos fatores sociodemográficos, só as habilitações literárias afetaram de forma estatisticamente significativa os resultados obtidos na dimensão "barreiras à atividade" $[F(2,228)=3,40 ; p=0,035]$ (Quadro II). Realizou-se um teste post-hoc que revelou não existirem diferenças estatisticamente significativas entre os grupos com escolaridade inferior ao $1 .^{\circ}$ ciclo e $10^{\circ}$ ciclo (DFM: 0,6; IC95\%: - 6,$7 ; 7,7)$, nem entre o grupo com escolaridade inferior ao $1 .{ }^{\circ}$ ciclo e superior ao $1 .{ }^{\circ}$ ciclo (DFM: 6,4; IC95\%: - 1,$1 ; 13,9)$. O grupo $1 .{ }^{\circ}$ ciclo apresentou um score médio mais alto em relação ao grupo com escolaridade superior ao $1 .^{\circ}$ ciclo (DFM: -5,8; IC95\%: -11,7; $-0,0)$.

Quanto à duração da doença, esta mostrou-se correlacionada de modo estatisticamente significativo com os resultados da dimensão "barreiras à atividade" $(r=0,17 ; p=0,010)$ (Quadro II).

Ainda em relação a esta dimensão, o grupo sem insulinoterapia apresentou uma média inferior à do grupo insulinotratado, sendo esta diferença estatisticamente significativa $[t(229)=-4,01$; DFM: $-12,9$; IC95\%: $-19,3 ;-6,6]$. O tratamento com insulina mostrou uma magnitude de efeito grande $(d=0,81)$. A hipoglicemia severa afetou os resultados das "barreiras à atividade"

\begin{tabular}{|c|c|c|c|}
\hline & $\beta$ & $t$ & $p$ \\
\hline \multicolumn{4}{|l|}{ Tensão psicológica } \\
\hline Constante & & 9,99 & $<0,001$ \\
\hline Género (feminino) & 0,18 & 2,74 & 0,007 \\
\hline Habilitações literárias ( $\leq 10^{\circ}$ ciclo) & $-0,15$ & $-2,35$ & 0,020 \\
\hline Hipoglicemia severa (sim) & 0,14 & 2,29 & 0,023 \\
\hline Complicações microvasculares (sim) & 0,19 & 3,05 & 0,003 \\
\hline \multicolumn{4}{|l|}{ Barreiras à atividade } \\
\hline Constante & & 24,02 & $<0,001$ \\
\hline Regime terapêutico (insulinoterapia) & 0,22 & 3,40 & 0,001 \\
\hline Hipoglicemia severa (sim) & 0,19 & 2,96 & 0,003 \\
\hline \multicolumn{4}{|l|}{ Alimentação desinibida } \\
\hline Constante & & 12,58 & $<0,001$ \\
\hline Habilitações literárias (< $1 .^{\circ}$ ciclo) & $-0,15$ & $-2,34$ & 0,020 \\
\hline Controlo glicémico (não controlado) & $-0,15$ & $-2,36$ & 0,019 \\
\hline
\end{tabular}

$\beta$ - Coeficiente de regressão estandardizado

$t$-t-student

$p-p$-value

DHP - Diabetes Health Profile Perfil de Saúde do Diabético

[ $t(229)=-3,64$; DFM: -14,1; IC95\%:-21,7; -6,5], com uma magnitude de efeito média $(d=0,75)$. Pacientes com complicações microvasculares apresentaram médias superiores nesta dimensão comparativamente aos utentes sem estas complicações $[t(229)=-2,67$; DFM: $-8,4$; IC95\%: -14,6; -2,2]. Não foram encontradas diferenças estatisticamente significativas entre os grupos das outras variáveis estudadas em relação a esta dimensão (Quadro II).

Quando inseridas num modelo considerando as cinco variáveis com impacto nas "barreiras à atividade", as habilitações literárias $(p=0,095)$, a duração da doença $(p=0,383)$ e as complicações microvasculares $(p=0,238)$ perderam a significância estatística (regime terapêutico $-p=0,008$; hipoglicemia severa $-p=0,013$ ). O novo modelo, criado apenas com o regime terapêutico $(p=0,001)$ e a hipoglicemia severa $(p=0,003)$, explica $9,0 \%$ da variabilidade desta dimensão $[F(2,228)=12,71$; $p<0,001 ; R^{2}{ }_{a d j}=0,09$ ] (Quadro III).

As habilitações literárias apresentaram relação estatisticamente significativa com a dimensão "alimentação desinibida" $[F(2,228)=3,35 ; p=0,037]$, com magnitude 
de efeito pequena $\left(\eta^{2}=0,03\right)$ (Quadro II). Entre o grupo com escolaridade inferior ao $1 .^{\circ}$ ciclo e superior ao $1 .^{\circ}$ ciclo existiu uma diferença estatisticamente significativa (DFM: 8,3; IC95\%: 0,3; 16,4), o que não aconteceu com as outras comparações (grupo com escolaridade inferior ao $1 .^{\circ}$ ciclo versus $1 .^{\circ}$ ciclo - DFM: 7,4; IC95\%: $-0,3 ; 15,1 ; 1 .{ }^{\circ}$ ciclo versus grupo com escolaridade superior ao $1 .{ }^{\circ}$ ciclo - DFM: 0,9; IC95\%: -5,4; 7,2).

A dimensão "alimentação desinibida" só demonstrou relação estatisticamente significativa com uma variável clínica, o controlo glicémico - os doentes não controlados apresentaram piores resultados, com uma média superior aos doentes controlados $[t(229)=2,23$; DFM: 5,7; IC95\%: 0,6; 10,7] com uma magnitude de efeito pequena $(d=0,33)$ (Quadro II).

As duas variáveis descritas mantiveram relação estatisticamente significativa com a "alimentação desinibida" quando consideradas conjuntamente (habilitações literárias $-p=0,020$; controlo glicémico $-p=0,019$ ). O modelo criado explica $4,0 \%$ da variância desta dimensão $\left[F(2,228)=5,27 ; p=0,006 ; R_{a d j}^{2}=0,04\right]$ (Quadro III).

\section{DISCUSSÃO}

Neste estudo, analisando de forma individual, para a dimensão "tensão psicológica", o género feminino (com uma magnitude de efeito pequena), menos habilitações literárias, ocorrência de hipoglicemia severa e a presença de complicações microvasculares (ambas com um efeito médio), teve um impacto negativo estatisticamente significativo na QoL, explicando $14 \%$ da variabilidade desta dimensão. Quanto à dimensão "barreiras à atividade", $9 \%$ da sua variabilidade reside na insulinoterapia e na ocorrência de hipoglicemia severa, ambas com uma magnitude de efeito grande. Os indivíduos com menor escolaridade e os não controlados do ponto de vista glicémico obtiveram uma pior QoL, de forma estatisticamente significativa, na dimensão "alimentação desinibida" e com uma magnitude de efeito pequena, explicando $4 \%$ da sua variabilidade.

Os valores médios das três dimensões, 20,0 para a "tensão psicológica", 30,5 para as "barreiras à atividade" e 27,7 para a "alimentação desinibida", foram ligeiramente inferiores aos reportados noutro estudo português com diabéticos tipo 2 (23,9, 33,4 e 33,9, respetivamente). ${ }^{16}$ Aparentemente, a população estudada nes- te trabalho terá uma maior QoL; no entanto, desconhecem-se os intervalos dos resultados no estudo previamente citado. A "tensão psicológica" mantém-se como o parâmetro menos afetado. Em relação às três dimensões do DHP, a correlação muito significativa entre todas revela coerência da escala.

Não se demonstrou uma relação estatisticamente significativa entre a QoL dos doentes diabéticos e a idade, contrariamente ao que ocorre na população geral, incluindo doentes com DM tipo $2,{ }^{12}$ provavelmente por ter sido utilizado um instrumento específico que avalia somente dimensões da QoL relevantes na DM.

O género feminino relacionou-se com um impacto negativo na dimensão "tensão psicológica", o que está de acordo com os dados encontrados por Schunk et $a l .{ }^{12} \mathrm{~A}$ maior prevalência de depressão e distúrbios afetivos e de ansiedade em mulheres, encontrada numa população de doentes diabéticos, pode ser uma explicação para este achado. ${ }^{23}$

Relativamente à proporção de mulheres e homens (1:1) encontrada, esta não coincide com os dados do OND referentes ao ano 2013 (2:3). ${ }^{2}$ Esta diferença pode ser explicada por ter sido utilizada uma amostra de conveniência e/ou por as mulheres serem geralmente mais frequentadoras de consultas médicas. ${ }^{24}$

Menor escolaridade mostrou relação estatisticamente significativa com menor QoL nas dimensões "tensão psicológica" e "alimentação desinibida". ${ }^{5-6}$ Os indivíduos com menor escolaridade apresentam, potencialmente, uma menor compreensão da doença e da terapêutica, sobretudo quando não existe adequação do discurso e dos materiais de apoio (folhetos, vídeos, livros) às habilitações literárias do doente, podendo acarretar alterações significativas na sua QoL.

A duração da doença apresentou, inicialmente, uma relação estatisticamente significativa com piores resultados nas "barreiras à atividade" mas, quando se consideraram possíveis viéses de confundimento (habilitações literárias, insulinoterapia, hipoglicemia severa e complicações microvasculares), esta relação perdeu-se. Estes dados são concordantes com a ausência de consenso sobre o impacto da duração da doença na QoL. ${ }^{5}$ Esta é uma medida de difícil avaliação (sujeita a vieses de registo e memória quando o diagnóstico não foi realizado pelo médico que regista e sim fornecida pelo doente) e cujo valor encontrado 
será sempre inferior ao real, uma vez que a doença se inicia até vários anos antes do diagnóstico que não são contabilizados.

Constatou-se que os doentes a realizar insulinoterapia apresentavam uma QoL menos satisfatória na dimensão "barreiras à atividade". Neves et al,${ }^{15}$ recorrendo ao DHP, compararam a QoL dos diabéticos, tipo $1 \mathrm{e}$ tipo 2, cujo regime terapêutico consistia na toma de antidiabéticos orais, insulinoterapia ou na combinação dos dois. Não sendo possível a confrontação direta de dados importa relevar que um menor nível de QoL estava associado a insulinoterapia e que, em todas as associações estudadas, a dimensão "barreiras à atividade" foi sempre afetada. ${ }^{15}$ Os mitos associados ao uso de insulina (maior probabilidade de complicações mais graves e morte precoce) aliada a automonitorização frequente (medições e perceção da variabilidade da glicemia de acordo com a alimentação) poderão ser aspetos que explicam esta menor QoL.

O número de fármacos não teve efeito na QoL, o que é consistente com o estudo de Tamir et al..$^{7}$ Cada vez mais existe uma maior preocupação do médico e da indústria farmacêutica em minimizar o impacto da ingestão de comprimidos na vida do doente e melhorar a adesão terapêutica. Estratégias como uso de comprimidos de dimensões reduzidas, associações fixas de fármacos, moléculas de efeito mais prolongado e redução do número de tomas são cada vez mais utilizadas. Do ponto de vista da QoL do doente, numa primeira fase será provavelmente mais benéfico aumentar o número de antidiabéticos orais do que iniciar insulinoterapia. ${ }^{7}$ No entanto, em doentes em que o controlo glicémico necessário à prevenção de complicações microvasculares crónicas só é alcançado com insulinoterapia, esta pode ter um papel benéfico na QoL. ${ }^{25}$ Cabe ao médico ponderar todas estas informações e envolver o doente na tomada de decisão. É também importante perceber porque se relaciona a insulinoterapia com pior QoL e se uma desmistificação deste regime terapêutico alteraria esta relação.

A ausência de controlo glicémico associa-se a QoL menos satisfatória, ${ }^{9}$ principalmente quando avaliada com instrumentos específicos. ${ }^{5}$ Neste trabalho, este parâmetro relacionou-se com valores mais elevados na dimensão "alimentação desinibida", o que seria expec- tável, já que hábitos alimentares inadequados levarão ao aumento da HbAlc. Por outro lado, se a doença não está controlada, é maior a probabilidade de ser necessário aumentar o número de fármacos, recorrer a insulinoterapia ou surgirem complicações microvasculares (estas duas últimas associadas também a menor QoL). Para uma classificação mais correta dos doentes controlados e não controlados recomendam-se estudos futuros que tenham em conta os objetivos individuais de HbAlc.

Os episódios de hipoglicemia severa são, por vezes, subestimados na DM tipo $2,{ }^{26}$ mas a sua avaliação é importante, verificando-se que $8,7 \%$ da amostra reportou um episódio destes no último ano e estes mostraram estar relacionados com valores mais elevados de "tensão psicológica" e "barreiras à atividade". A relação destes episódios com QoL menos satisfatória é concordante com estudos anteriores. ${ }^{6,10} \mathrm{O}$ receio de recorrência de episódios de hipoglicemia e das suas consequências (acidentes, coma e mesmo morte) poderá levar a ansiedade, medo e condicionamento das atividades de vida diária e consequentemente à diminuição da autonomia dos doentes com DM.

Tal como descrito por Boini et al, ${ }^{6}$ as complicações microvasculares apresentaram uma relação estatisticamente significativa com pior QoL, ao contrário das macrovasculares. Os doentes com sequelas mais graves serem seguidos fundamentalmente ao nível hospitalar e a impossibilidade dos mesmos se dirigirem à USF, devido às limitações impostas pela doença, poderão justificar este achado. Também o facto das complicações microvasculares serem geralmente progressivas, com possivelmente maior condicionamento da vida do doente, como por exemplo no caso da cegueira ou da insuficiência renal terminal, poderá contribuir para este resultado. As complicações macrovasculares ocorrem de forma episódica (acidente vascular cerebral ou enfarte agudo do miocárdio) e poderão estar associadas a sequelas ou não.

O uso de instrumentos específicos para a avaliação da QoL em doentes diabéticos é uma mais-valia porque podem apresentar maior sensibilidade na avaliação de domínios com particular relevância para estes doentes e não ser tão influenciados por variáveis não relacionadas com a DM, como o emprego ou finanças. ${ }^{27} \mathrm{No}$ entanto, nenhuma ferramenta conseguiu ainda avaliar 
todos os domínios relevantes na QoL dos doentes com DM, existindo diversas e que deverão ser aplicadas de acordo com o que se pretende avaliar (e.g., os instrumentos "Crenças em Saúde Relacionadas com a Diabetes", "Escala de Medição do Impacto da Diabetes", "DHP", "Escala de Bem-Estar Psicológico"). ${ }^{28}$

O tamanho amostral considerável e o facto de todas as entrevistas terem sido realizadas pela mesma investigadora são também pontos fortes deste estudo.

As diferenças substanciais no número de elementos de alguns grupos foi uma limitação, pelo que as conclusões deverão ser cuidadosamente analisadas. Poderão existir associações estatisticamente significativas que não foram possíveis de ser demonstradas por causa da insuficiência do tamanho de cada grupo. Por outro lado, o tipo de agregação de grupos poderá ter criado ou mascarado associações. Verificou-se que esta última medida não resolveu totalmente o problema da diferença de tamanho amostral, o que impossibilitou uma avaliação mais detalhada. Seria importante realizar estudos com amostras em clusters para assegurar grupos dimensionalmente semelhantes, permitindo uma análise mais completa e correta.

Tendo recorrido a algumas questões sociodemográficas e clínicas e a um questionário de autopreenchimento, não se podem ignorar os possíveis vieses de memória e as dificuldades de interpretação. No entanto, 0 facto destes dados terem sido colhidos pela mesma investigadora e existir a possibilidade dos doentes tirarem dúvidas poderá ter minimizado estes potenciais vieses. A informação clínica foi acedida através do SAM, com possíveis erros de registo ou codificação.

Devido à natureza transversal do estudo não é possível retirar ilações de causa-efeito entre as variáveis e as dimensões da QoL avaliadas.

Por limitações de tempo e custos recorreu-se a uma amostra de conveniência, que poderá não ser representativa da população. A escolha de doentes vigiados e com um registo de $\mathrm{HbAlc}$ no ano de 2013 poderá também ser uma limitação, uma vez que um doente mais utilizador dos serviços de saúde teve maior probabilidade de participar no estudo.

Os critérios de exclusão constituíram um viés de seleção. Doentes com doença mais avançada, maior número de complicações, nomeadamente retinopatia, podem ter sido impossibilitados de participar por in- capacidade de ler e responder ao questionário. Assim, os valores das dimensões que pretendem avaliar a QoL podem estar subestimados.

Os modelos criados com as variáveis estudadas explicam uma pequena parte da variância da QoL dos doentes com DM tipo 2 desta amostra, o que poderá ser justificado pela complexidade desta avaliação. É expectável que sejam muitos os fatores que contribuam para a variação da QoL. Os futuros estudos deverão debruçar-se noutros parâmetros que possam afetar a QoL, nomeadamente os hábitos e estilos de vida, parâmetros antropométricos, outros valores analíticos para além da HbAlc e a existência de co-morbilidades. No entanto, para além de perceber o que afeta a QoL, é fundamental perceber que estratégias se podem criar para minimizar o impacto da doença no quotidiano.

Os profissionais de saúde devem, por isso, prestar maior atenção aos doentes com estas características, tentando minimizar o efeito negativo da doença no seu dia-a-dia. Perceber quais as determinantes da QoL das pessoas com DM tipo 2 é um passo primordial para uma medicina centrada no doente, procurando-se um equilíbrio entre os imperativos do controlo da doença e as necessidades, desejos e expectativas do paciente em relação à doença e ao futuro, encarando a QoL como um objetivo terapêutico.

\section{REFERÊNCIAS BIBLIOGRÁFICAS}

1. International Diabetes Federation. IDF diabetes atlas. 6 th ed. Brussels: IDF; 2013. ISBN 2930229853

2. Sociedade Portuguesa de Diabetologia. Diabetes: factos e números 2014 - Relatório anual do Observatório Nacional da Diabetes. Lisboa: SPD; 2014.

3. Krumholz HM. Outcomes research: generating evidence for best practice and policies. Circulation. 2008;118(3):309-18.

4. WHOQOL-Group.WHOQOL: measuring quality of life. Geneva:World Health Organization; 1997.

5. Rubin RR, Peyrot M. Quality of life and diabetes. Diabetes Metab Res Rev. 1999;15(3):205-18.

6. Boini S, Erpelding ML, Fagot-Campagna A, Mesbach M, Chwalow J, Penfornis $A$, et al. Factors associated with psychological and behavioral functioning in people with type 2 diabetes living in France. Health Qual Life Outcomes. 2010;8:124.

7. Tamir O, Wainstein J, Raz I, Shemer J, Heymann A. Quality of life and patient-perceived difficulties in the treatment of type 2 diabetes. Rev Diabet Stud. 2012;9(1):46-54.

8. Zhang P, Brown MB, Bilik D, Ackermann RT, Li R, Herman WH. Health utility scores for people with type 2 diabetes in U.S. managed care health plans. Diabetes Care. 2012;35(11):2250-6. 
9. Kamarul Imran M, Ismail AA, Naing L, Wan Nohamad WB. Type 2 diabetes mellitus patients with poor glycaemic control have lower quality of life scores as measured by the Short Form-36. Singapore Med J. 2010;51(2):157-62.

10. Alvarez-Guisasola F, Yin DD, Nocea G, Qiu Y, Mavros P. Association of hypoglycemic symptoms with patients' rating of their health-related quality of life state: a cross sectional study. Health Qual Life Outcomes. 2010;8:86.

11. Kleefstra N, Landman GW, Houweling ST, Ubink-Veltmaat LJ, Logtenberg SJ, Meyboom-de Jong B, et al. Prediction of mortality in type 2 diabetes from health-related quality of life (ZODIAC-4). Diabetes Care. 2008;31(5):932-3.

12. Schunk M, Reitmeir P, Schipf S, Völzke H, Meisinger C, Thorand B, et al. Health-related quality of life in subjects with and without Type 2 diabetes: pooled analysis of five population-based surveys in Germany. Diabet Med. 2012;29(5):646-53.

13. Lopes I, Correia S, Marques L, Couto L. Qualidade de vida das pessoas com diabetes mellitus tipo 1. Rev Port Diabetes. 2010;5(3):110-2.

14. Torres A, Fonte M, Martins V, Silva I, Cardoso H, Rocha C, et al. Qualidade de vida: estudo de uma população com diabetes mellitus tipo 1 [Quality of life: a study in a population with type II diabetes]. Rev Port Endocrinol Diab Metab. 2010;5(2):49-54. Portuguese

15. Neves C, Carvalheiro M, Ferreira PL. Qualidade de vida em pessoas com diabetes mellitus [Quality of life in people with diabetes mellitus]. Arquivos Med. 2002;16(4/5/6):200-10. Portuguese

16. Ferreira MM, Veríssimo MT. Melhoria contínua da qualidade na prestação de cuidados de saúde ao doente diabético tipo 2 [Health care quality of life improvement in type 2 diabetic patients]. Rev Port Saúde Pública. 2008;26(2):37-60. Portuguese

17. Ferreira PL, Neves C. O perfil de saúde do diabético [The diabetes health profile]. Bol Soc Port Diabetol. 2002;6:10-7. Portuguese

18. American Diabetes Association Workgroup on Hypoglycemia. Defining and reporting hypoglycemia in diabetes: a report from the American Diabetes Association Workgroup on Hypoglycemia. Diabetes Care. 2005;28(5):1245-9.

19. American Diabetes Association. Executive summary: standards of medical care in diabetes - 2013. Diabetes Care. 2013;36 Suppl 1:S4-10.

20. Fowler MJ. Microvascular and macrovascular complications of diabetes. Clin Diabetes. 2008;26(2):77-82.

21. Garson GD. Testing statistical assumptions. Asheboro: Statistical Associates Publishing; 2012.

22. Cohen J. Statistical power analysis for the behavioral sciences. 2nd ed. Hillsdale, NJ: Routledge; 1988. ISBN 9780805802832
23. Hermanns N, Kulzer B, Krichbaum M, Kubiak T, Haak T. Affective and anxiety disorders in a German sample of diabetic patients: prevalence, comorbidity and risk factors. Diabet Med. 2005;22(3):293-300.

24. Vintém JM, Guerreiro MD, Carvalho H. Desigualdades de género e sociais na saúde e doença em Portugal: uma análise do módulo "Saúde" do European Social Survey - 2004. In VI Congresso Português de Sociologia - Mundos sociais: saberes e práticas, Faculdade de Ciências Sociais e Humanas (UNL), 25-28 de junho de 2008.

25. Pouwer F, Hermanns N. Insulin therapy and quality of life: a review. Diabetes Metab Res Rev. 2009;25 Suppl 1:S4-10.

26. Zammitt NN, Frier BM. Hypoglycemia in type 2 diabetes: pathophysiology, frequency, and effects of different treatment modalities. Diabetes Care. 2005;28(12):2948-61.

27. Speight J, Reaney MD, Barnard KD. Not all roads lead to Rome: a review of quality of life measurement in adults with diabetes. Diabet Med. 2009;26(4):315-27.

28. Ferreira PL, Neves C. Qualidade de vida e diabetes [Quality of life and diabetes]. Rev Port Clin Geral 2002;18(6):402-8. Portuguese

\section{CONFLITO DE INTERESSES}

A autora Carla Lopes da Mota declara ser editora da RPMGF não tendo participado no processo editorial deste artigo.

\section{PRÉMIOS}

O trabalho final foi vencedor do Prémio Ernesto Roma Cuidados de Saúde Primários 2013.

\section{COMISSÃO DE ÉTICA}

Estudo realizado após parecer favorável da Comissão de Ética da Saúde da ARS Norte.

\section{ENDEREÇO PARA CORRESPONDÊNCIA}

Carla Lopes da Mota

Rua Delfim Ferreira, 231 - $1^{\circ}$ Dto Frt, 4410-436 Arcozelo

TLM 964780620

carlalopesmota@gmail.com

Recebido em 26-08-2014

Aceite para publicação em 22-05-2015

Artigo escrito ao abrigo do novo acordo ortográfico. 


\section{ABSTRACT}

\section{QUALITY OF LIFE AND ASSOCIATED FACTORS IN TYPE 2 DIABETES MELLITUS: A CROSS-SECTIONAL STUDY}

Introduction: Type 2 diabetes mellitus (DM) has high social and economic costs. It is often associated with decreased quality of life (QoL).

Objectives: To assess the impact of demographic and clinical factors on QoL in a sample of patients with DM type 2.

Study type: Cross-sectional study.

Setting: Espinho FHU.

Population: Patients with DM type 2 followed by the Espinho Family Health Unit in December of 2012.

Methods: A convenience sample of 231 patients visiting the FHU for a DM consultation was selected. Demographic and clinical data were collected and the Diabetic Health Profile questionnaire was applied to evaluate QoL. The association of continuous variables with DHP dimensions was assessed using the Pearson correlation coefficient and the influence of categorical variables was tested with the Student t-test and ANOVA.

Results: Female gender, lower educational level, severe hypoglycemia, and microvascular complications had a negative impact on the 'psychological distress' dimension of QoL. 'Barriers to activity' were negatively associated with insulin therapy and severe hypoglycemia. Patients with lower educational level and without glycemic control had lower QoL with regard to 'disinhibited eating'.

Conclusions: QoL is a complex concept with multiple determinants. It is important to study these factors and assess them in each patient with DM for more effective intervention.

Keywords: Type 2 Diabetes Mellitus; Quality of Life. 